two per week; 4 patients consumed a takeaway every other day and 1 patient consumed 15 takeaways in total. There was no clear distribution between acute and rehabilitation wards and there were no obvious gender differences. The mean number of takeaways per ordering patient was five. At least half of the takeaways were curries.

The wide range of cost and high average cost probably reflected group bookings registered to a patient and so underestimated the total number of takeaways. This was confirmed at a unit meeting with patient representatives who felt that a takeaway should on average cost around $£ 5$ and that sharing takeaways or group bookings registered to a patient occurred frequently. If extrapolated over a year, a patient would spend on average $f 727$ on takeaways. The annual cost for all patients in the unit would be $£ 47423$.

Possible ways of reducing 'excessive takeaways,' though this amount is undefined, include individual care plans or designated 'takeaway nights,' which is the current policy within the learning disability directorate. Although the average number of takeaways per patient was five within a 21-day period and could potentially increase to six if takeaways were ordered twice a week, as per the learning disability model, it was felt that the average number of takeaways calculated was a gross underestimation. An outright ban could be enforced on security grounds. The issue of restricting patient choice, patient autonomy, poor-quality hospital food and infringement on human rights have been raised as counterarguments.

1 Osborn DP, Levy G, Nazareth I, Petersen I, Islam A, King MB. Relative risk of cardiovascular and cancer mortality in people with severe mental illness from the United Kingdom's general practice research database. Arch Gen Psychiatry 2007; 64: 242-9.

2 Lawrence DM, Holman CDJ, JablenskyAV, Hobbs MST. Death rate from ischaemic heart disease inWestern Australian psychiatric patients 1980-1998. BrJ Psychiatry 2003; 182: 31-6.

3 Mackin P, Bishop D, Watkinson H, Gallagher P, Ferrier IN. Metabolic disease and cardiovascular risk in people treated with antipsychotics in the community. BrJ Psychiatry 2007; 191: 23-9.

Yasir Kasmi Specialist Registrar in Forensic Psychiatry, Newton Lodge, Yorkshire Centre for Forensic Psychiatry, Ouchthorpe Lane, Wakefield WF1 3SP, email: yasirkasmi@hotmail.com

doi: $10.1192 / p b .33 .7 .276 a$

\section{Injectable opioid prescribing in Oxfordshire}

We sought to replicate in the Specialist Community Addiction Service that covers the county of Oxfordshire the audit on the prescribing of injectable opiates undertaken by White \& Shearman in Cornwall. ${ }^{1}$ We identified 19 patients (14 males and 5 females) on regular injectable opioid prescriptions: 10 on diamorphine $(53 \%), 5$ on methadone (26\%), 2 on pethidine (11\%) and 1 on morphine (5\%); 1 person dropped out of the service and was not further included in the study. Of these, 17 were interviewed using a slightly modified version of the audit tool kindly provided by White \& Shearman. Only one person had been started on a script in the past 4 years while others had been on this type of treatment for an average of 9.5 years (s.d. = 4.1). Three patients, all females, had been dependent on prescribed injectable opioid analgesics. The other 14 had been heroin users for an average of 15.8 years (s.d. $=6.3$ ) before being started on an injectable prescription. Compared with Cornwall, our audit reveals an older group of users who had been started on injectable scripts after lengthy periods of oral substitution treatment (average 9.2 years, s.d. =6.1). The Oxfordshire cohort was also relatively more stable with no reports of overdoses while using the prescribed drug or additional opiate use in the previous month. Alcohol consumption was also low, with only two clients reporting problematic levels of drinking. When asked, $47 \%$ said they had no intention to ever come off the script; $29 \%$ would consider it in 5 years and $24 \%$ in 1 year. The clients, regardless of the drug injected, were approximately evenly split between almost exclusive intramuscular or intravenous use with little crossover. Around half the patients reported experiencing any harmful physical consequences from prescribed injectable opiates. Occasional abscesses were the main problem faced by those injecting intramuscularly, whereas those who practised intravenous injections reported abscesses, deep vein thrombosis and cellulitis. All had found access to medical care when needed. The overwhelming majority claimed to consistently use clean needles but the answers were more equivocal with regard to sterile injecting technique. Direct supervision of injecting techniques was minimal with only two clients $(11 \%)$ remembering having been observed on one occasion by their general practitioner or another health professional. In conclusion, we found interesting similarities between the client groups in Oxford and Cornwall, which might indicate that a clinical rationale for providing injectable opiates to a niche population exists and that it transcends geographical and social regional differences.

\section{White R, Shearman L. Injectable opiate prescribing in Cornwall. Psychiatr Bull 2008; 32 : 387-90.}

*Joseph El-Khoury Specialty Registrar in Adult Psychiatry, Oxfordshire and Buckinghamshire Mental Health NHS FoundationTrust, Oxford
Specialist CommunityAddiction Service, The Rectory Centre, Rectory Road, Oxford OX4 1DU, email: Josephelkhoury@doctors.org.uk, Andrew McBride Consultant in Addiction Psychiatry, Oxfordshire and Buckinghamshire Mental Health NHS Foundation NHS Trust, The Rectory Centre, Oxford

doi: 10.1192/pb.33.7.277

\section{Prevalence of challenging behaviour among older in-patients: a pilot study}

Older people occupy two-thirds of in-patient beds in acute general hospitals Pre-existing mental health disorders are independent predictors of poor outcomes such as increased mortality and length of stay, loss of independent function and higher rates of institutionalisation. ${ }^{1}$ Disruptive behaviour can have a negative effect on the nursing and other healthcare staff and can affect the quality of care provided to other patients on the ward. ${ }^{2}$ There are no published studies on the prevalence of behavioural problems in older in-patients in the UK

We conducted a pilot study to determine the prevalence of challenging behaviour in older people on two care of the elderly wards in an acute general hospital. Patients aged 65 years and older were included. The Crichton Royal Behavioural Rating Scale (CRBRS) ${ }^{3}$ was used to identify patients with challenging behaviour. The CRBRS is a descriptive scale designed to assess patients on psychogeriatric wards. The main scale items are mobility, orientation, communication, cooperation, restlessness, dressing, feeding, mood and continence. Each modality has a score of between 1 and 5 , where 1 is normal and 5 is the most abnormal. The scores for cooperation, restlessness and sleep are those that provide information on the prevalence of challenging behaviour. Patients with a score of 2 or more for restlessness or sleep, or 4 or more for cooperation are considered to have a challenging behaviour.

The scale was completed separately for daytime and night-time by interviewing nursing staff completing the respective shifts. Medical notes were examined to identify any previous mental health problems and to determine whether the patients were on psychiatric medication. The study was approved by the trust's clinical governance department.

In total, 58 patients were studied (47 males). Mean age was 81 years (range 66-96). Challenging behaviour was identified in 16 patients (29\%) according to the CRBRS criteria: 5 scored for restlessness only; 3 for restlessness, sleep disturbance and cooperation; 1 scored for sleep disturbance and cooperation, and 1 for 
sleep disturbance only. Of the 58 patients studied, 21 had a known psychiatric history before admission (14 dementia, 6 depression, 1 schizophrenia). Of the 16 patients with challenging behaviour, 8 had dementia and 1 had schizophrenia; 6 patients did not have a history of a psychiatric disorder. A history of dementia was significantly more common among patients with challenging behaviour $(P=0.008)$. Aggressive behaviour was seen in $48 \%$ of patients in this group and was not seen among patients not scored to have challenging behaviour. Wandering was seen in $25 \%$ of patients with challenging behaviour.
We conclude that a significant number of elderly patients admitted to our hospital had challenging behaviour Dementia seemed to be associated with challenging behaviour, and aggressive behaviour was seen in a significantly large proportion of patients. A larger study is warranted.

Working Group for the Faculty of Old Age Psychiatry. Who Cares Wins. Improving the Outcome for Older PeopleAdmitted to the General Hospital: Guideline for the Development of Liaison Mental Health Services for Older People. Royal College of Psychiatrists, 2005
2 Sourial R, McCusker J, Cole M, Abrahamowicz M. Agitation in demented patients in an acute care hospital: prevalence, disruptiveness and staff burden. Int Psychogeriatr 2001; 13 183-97.

3 Robinson RS. The organisation of a diagnostic and treatment unit for the aged in a mental hospital. In Psychiatric Disorders in the Aged: 186-205. Geigy, 1965.

Anoja Rajapakse Medical Division, Newark Hospital, Sherwood Forest NHS Trust, Boundary Road, Newark NG24 4DE, email: anoja.ilangas@ gmail.com, Jacqui Bussin Whiston and St Helens Hospitals, St Helens and Knowsley NHS Trust, UK

doi: $10.1192 / \mathrm{pb} .33 .7 .277 \mathrm{a}$ 